\title{
Planificacion de tratamiento con software para cirugía guiada en implantologia oral
}

\author{
Treatment planning with software for \\ guided surgery in implant dentistry
}

\author{
Alexandre Oliveira $N^{*}$, Matos Garrido $N^{* *}$, España López $A^{* *}$, Jiménez Guerra $A^{* *}$, \\ Ortiz García I**, Velasco Ortega E***
}

\section{RESUMEN}

La planificación preoperatoria de la posición de los implantes constituye una parte importante de la coordinación quirúrgica y prostodóncica que se ha ido incrementando para conseguir resultados funcionales y estéticos. La cirugía guiada de implantes ha aumentado su popularidad, particularmente debido a los avances y a la utilización de la tomografia computarizada de haz cónico (CBCT) y a la planificación del tratamiento con implantes mediante programas informáticos 3D que valoran la localización del implante. De hecho, los pacientes edéntulos pueden ser diagnosticados con una CBCT y tratados con varios implantes por cirugía guiada y carga inmediata. La CBCT constituye un método no invasivo para describir las estructuras maxilofaciales evaluando la cantidad y calidad ósea de los rebordes alveolares. La incorporación de softwares específicos de implantología guiada puede mejorar la planificación virtual de la cirugía sin colgajo y los resultados de los implantes colocados con una férula quirúrgica guiada. En muchos casos, la carga inmediata optimiza el éxito de la técnica de cirugía guiada con muchos beneficios como la reducción del tiempo del tratamiento y el confort del paciente.

PALABRAS CLAVE: Implantología oral guiada, tomografía de haz cónico, programa informático, carga inmediata, implantes dentales.

\section{ABSTRACT}

Preoperative planning of the implant position as part of a coordinated prosthetic and surgical concept is becoming increasingly important regarding function and esthetics. Guided implant surgery is increasing in popularity, particularly due to advances and increased usage of cone beam computed tomography $(\mathrm{CBCT})$ and dental implant treatment planning software allowing three-dimensional assessment of the implant site. In fact, edentulous patients can be diagnosed by a CBCT and treated with several implants for rehabilitation with guided surgery and immediate loading. The CBCT provides a noninvasive method to describe maxillofacial structures and assess bone volume and density of alveolar ridges. The introduction of specific softwares of guided implant dentistry can improve the virtual planning of flapless surgery and outcomes of dental implant placed in edentulous alveolar ridges by template guided surgery. In many cases, the immediate-loading protocol maximises the sucess of the guided surgery techniques with many benefits, such as short time and maximum patient comfort.

* $\quad$ Licenciado en Odontología. Máster en Implantología Oral. Universidad de Sevilla. Práctica privada en Coimbra.

** Profesor Colaborador Docente de Odontología Integrada de Adultos y Gerodontología. Facultad de

Odontología. Profesor del Máster de Implantología Oral. Universidad de Sevilla.

*** Profesor Titular de Odontología Integrada de Adultos y Gerodontología. Facultad de Odontología.

Director del Máster de Implantología Oral. Universidad de Sevilla. 
KEY WORDS: Guided-implant dentistry, cone beam tomography, software, immediate loading, dental implants.

Fecha de recepción: 19 de febrero de 2019.

Fecha de aceptación: 25 de febrero de 2019.

Alexandre Oliveira N, Matos Garrido N, España López A, Jiménez Guerra A, Ortiz García I, Velasco Ortega E. Planificacion de tratamiento con software para cirugía guiada en implantologia oral.

2019; 35, (2): 59-68

\section{INTRODUCCIÓN}

El tratamiento con implantes dentales representa, en la actualidad, una opción terapéutica muy exitosa en la práctica clínica odontológica. La rehabilitación con implantes, tras la evaluación sistémica y oral del paciente, la determinación del plan de tratamiento y un correcto protocolo quirúrgico y prostodóncico, constituye una visión multidisciplinaria del tratamiento implantológico, agregando cada vez más soluciones y herramientas que ayudan al clínico a tomar mejores decisiones, y a conseguir las rehabilitaciones orales más predecibles, seguras y consistentes ${ }^{1}$.

Le evolución de los softwares o programas informáticos permiten la captación de información de diferentes fuentes, hacer su procesamiento y ponerla a la disposición de los clínicos. Los programas informáticos, que inicialmente tenían un papel más de apoyo y de asistencia a la visualización de las imágenes radiológicas, actualmente procesan archivos que generan guías para usar en medios complementarios de diagnóstico $\mathrm{y}$ en el procedimiento quirúrgico, todo a través de procesos de elaboración virtual ${ }^{2-4}$.

Con el crecimiento de la técnica de implantología oral asistida por ordenador, fue necesario desarrollar mecanismos de seguridad y de precisión, para conseguir una técnica viable y precisa. Los resultados exitosos fueran continuadamente basados en la evidencia clínica y reflejado en la bibliografía. La colocación de implantes provisionales en boca para obtener puntos de referencia en arcadas edéntulas, el control de la orientación de los cortes axiales al plano oclusal, las técnicas de segmentación digital, todas estas funcionalidades juntas contribuyen a mejorar la técnica y consecuentemente el resultado final ${ }^{5-6}$.
El resultado de todo el flujo de trabajo, desde la planificación a la cirugía se queda reflejado en la guía quirúrgica. Su precisión es un factor fundamental para evaluar el éxito la rehabilitación, y comprobar que los sistemas de cirugía guiada, presenta un grado importante de precisión ${ }^{7}$. Además, el resultado final de una rehabilitación pasa por la evaluación de las tasas de supervivencia de los implantes utilizados y los resultados prostodóncicos, que puede reflejar la fiabilidad de estos sistemas ${ }^{8}$.

\section{LA TOMOGRAFIA COMPUTARIZADA DE HAZ CONICO}

Con la aparición de la tomografía computarizada de haz cónico (en inglés CBCT), se ha revolucionado el diagnóstico en implantología oral, y también en el tratamiento con la técnica de cirugía guiada asistida por ordenador ${ }^{9-10}$.

La CBCT reduce de forma importante la exposición a la radiación, presentando una mejor calidad de las imágenes. Permite al clínico generar múltiples cortes tomográficos en diversos planos de una región anatómica que se pretenda estudiar, utilizando un haz de rayo rotacional en forma cónica, y mediante una serie de algoritmos matemáticos, obtener múltiples proyecciones con una sólo rotación, haciendo de esta forma la reconstrucción de las estructuras anatómicas en una imagen 3D (Figuras 1 y 2) ${ }^{9}$. En combinación con el software de planificación de implantes, el uso de imágenes de CBCT permite planificar virtualmente la posición óptima de un implante con respecto a las estructuras anatómicas vecinas y las futuras necesidades protésicas (Figuras 3-6) ${ }^{11}$.

La adquisición de la CBCT permite que el paciente pueda estar sentado o de pie, y la fuente de rayos 


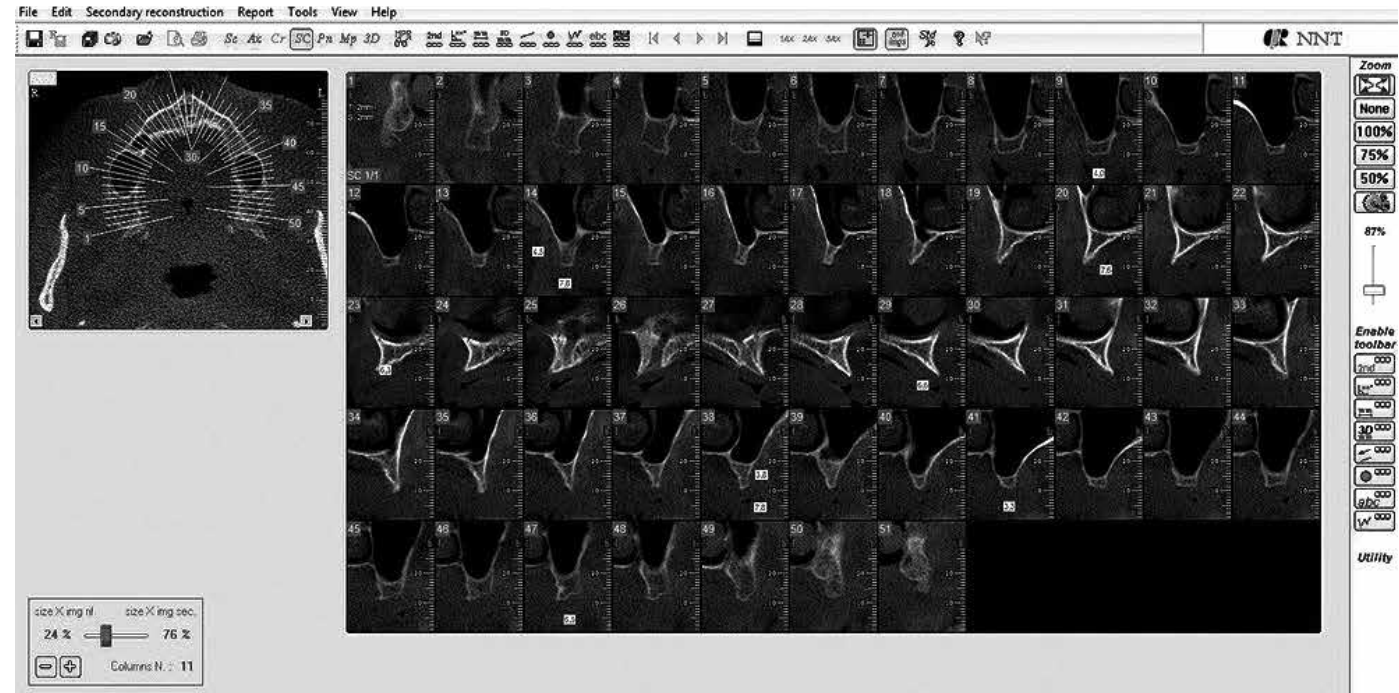

Figura 1. Reconstrucción de imágenes de tomografia computarizada de haz cónico en maxilar superior (CBCT).

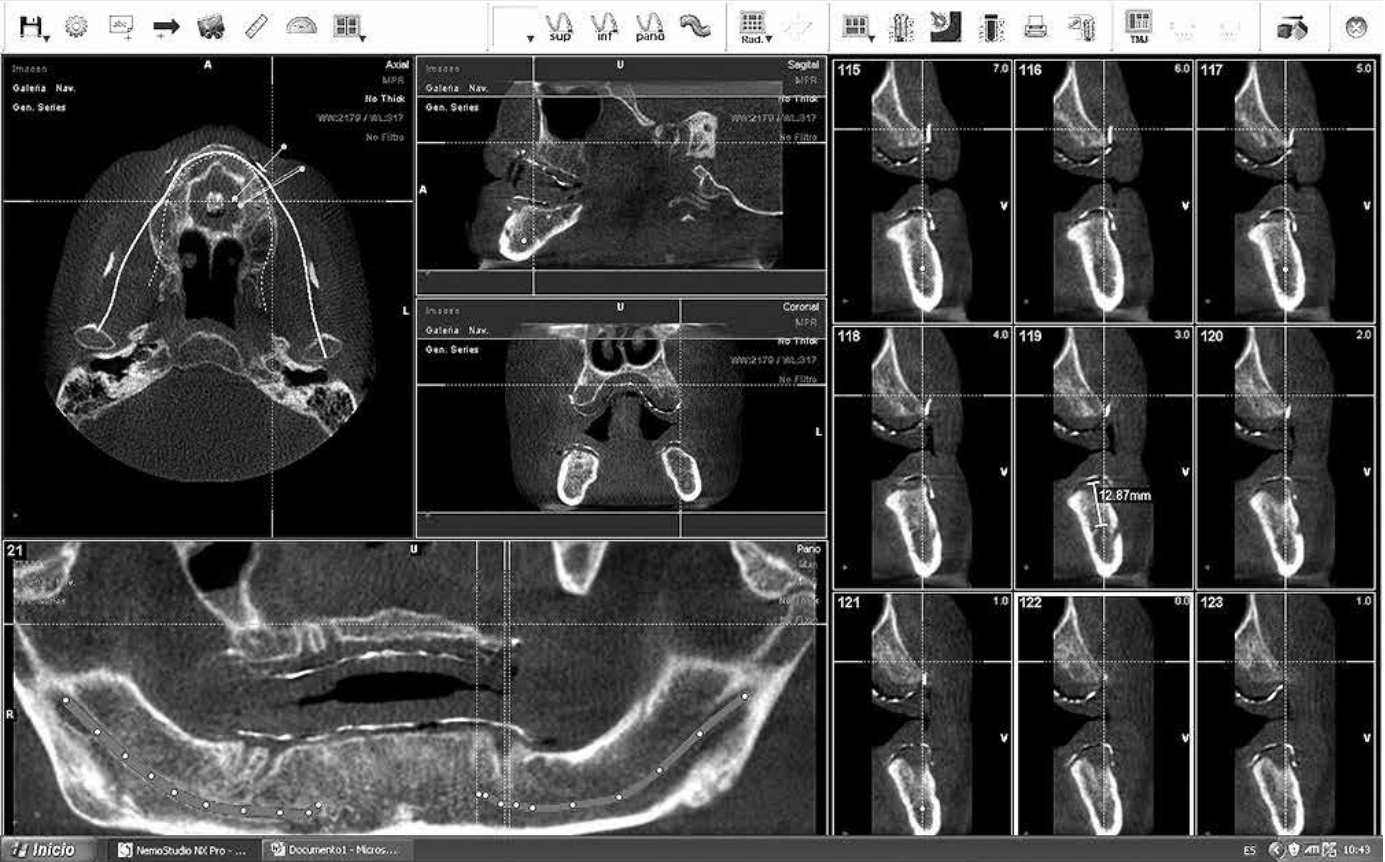

Figura 2. Reconstrucción de imágenes de tomografia computarizada de haz cónico en mandíbula (CBCT). de los dientes con restauraciones de este tipo 10. Sin embargo, la utilización de imágenes de CBCT puede ser muy útil en la localización de estructuras anatómicas importantes para la planificación de la técnica quirúrgica, como por ejemplo los vasos sanguíneos de la pared lateral del seno maxilar, la presencia de septos intrasinusales, vasos sanguíneos presentes en la sínfisis mandibular, y visualización de la posición del conducto del nervio dentario inferior (Figuras 1 y 2$)^{10}$.

La CBCT permite valorar adecuadamente las características anatómicas del maxilar superior y de la mandíbula para la planificación de la cirugía de implantes (Figuras $1 \mathrm{y}$ 2) ${ }^{12-13}$. En el maxilar superior, la CBCT puede identificar un reborde alveolar insuficiente para la colocación de los implantes de $4 \mathrm{~mm}$ de diámetro. Esta información sugerirá la necesidad de realizar

rota $360^{\circ}$ alrededor de su cabeza, obteniendo múltiples imágenes. Seguidamente el programa informático archiva todas las imágenes, que se componen de unidades básicas digitales (voxels). Las imágenes presentan un mayor grado de precisión que el TC, y tienen un menor coste económico ${ }^{9}$.

El paciente debe estar inmóvil durante la adquisición para alcanzar una buena imagen. De lo contrario, la imagen puede mostrar defectos. También habrá artefactos en la imagen alrededor de las prótesis metálicas, dificultando la evaluación técnicas de aumento de volumen óseo, de expansión ósea con osteotomos o la inserción de implantes de menor diámetro (Figura 1) 9-10,12. En la mandíbula, la CBCT puede obtener imágenes de determinados accidentes anatómicos como la concavidad lingual en la sínfisis. A veces, la mandíbula presenta una concavidad lingual en su región posterior que puede ser también diagnosticada. La CBCT puede identificar el conducto dentario inferior y valorar si es único o está dividido o la situación bucolingual del mismo, lo que es realmente importante para la cirugía de implantes (Figura 2) 9-10,13. 
Además del volumen del hueso alveolar, las condiciones locales específicas durante una cirugía guiada de implantes, como la densidad ósea de la zona elegida, pueden cambiar las dimensiones previstas de la osteotomía, debido a una mala evaluación de los exámenes de diagnóstico y percibidas objetivamente sólo durante la cirugía ${ }^{11}$.

La densidad del hueso receptor tiene un papel importante en la estabilidad primaria de los implantes y en la predictibilidad del éxito de la oseointegración y posterior rehabilitación inmediata. Un implante colocado en un hueso compacto tiene una mayor probabilidad de obtener estabilidad primaria y soportar las fuerzas inmediatas iniciales. Se puede encontrar este tipo de hueso en la región interforaminal de la mandíbula. El hueso cortical laminar cicatriza con poca formación de hueso nuevo, originando hueso más denso. Su porosidad fina, no superior al $10 \%$ del total, favorece una mejor interconexión mecánica en comparación con el hueso trabecular, lo que puede llegar a $80-95 \%$ de porosidad ${ }^{14}$.

\section{PLANIFICACION DIGITAL DE CIRUIGÍA GUIADA}

La colocación de implantes a través de la técnica convencional presenta algunas dificultades como el movimiento del paciente durante la cirugía, la necesidad de usar una mayor anestesia local por el aumento del tiempo necesario para terminar la cirugía, una visualización disminuida del campo operatorio, la transferencia mental a veces difícil de la imagen del examen radiográfico bidimensional al ambiente quirúrgico tridimensional, y las limitaciones protésicas que un caso clínico puede tener por una mala colocación del implante. Así, durante un período de tiempo limitado y con una visión restringida, el cirujano tiene que tomar diversas decisiones mientras que trata un paciente consciente, bajo condiciones asépticas. Por lo tanto, una planificación preoperatoria cuidadosa del número de implantes que se colocarán, su tamaño, su posición, y su inclinación favorecerá la labor del cirujano, permitiendo concentrarse en el paciente y en el campo operatorio que maneja ${ }^{15-16}$.

La utilización de la técnica de cirugía guiada asistida por ordenador permite de una forma práctica incluir la prótesis final como factor de decisión en la planificación de la colocación de los implantes. Virtual-

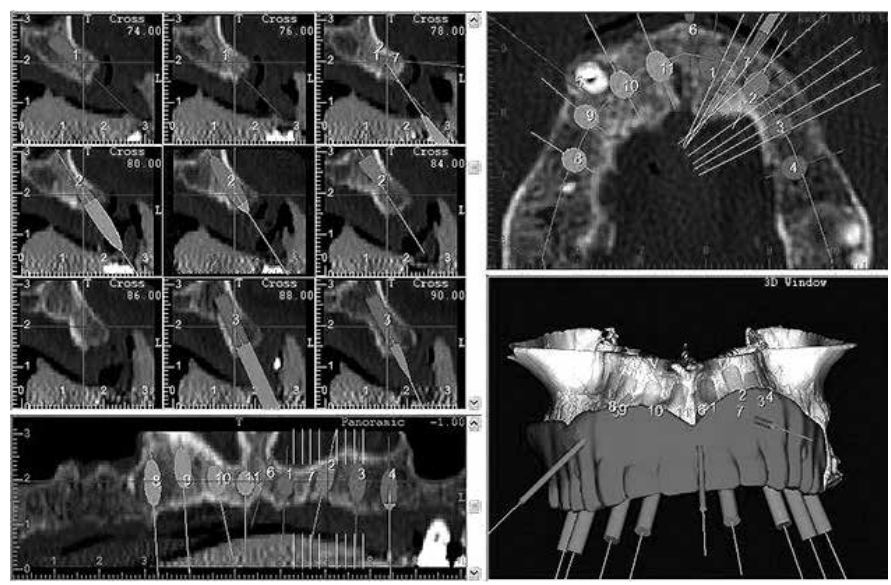

Figura 3. Planificación quirúrgica con software Dental Slice ( ) de imagen de CBCT.
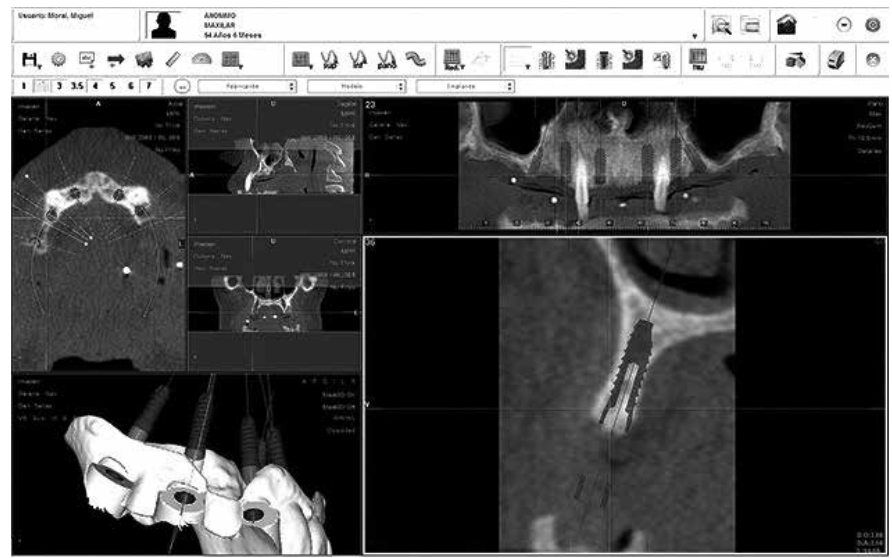

Figura 4. Planificación quirúrgica con software Nemoscan ${ }^{\circledR}$ de imagen de CBCT.

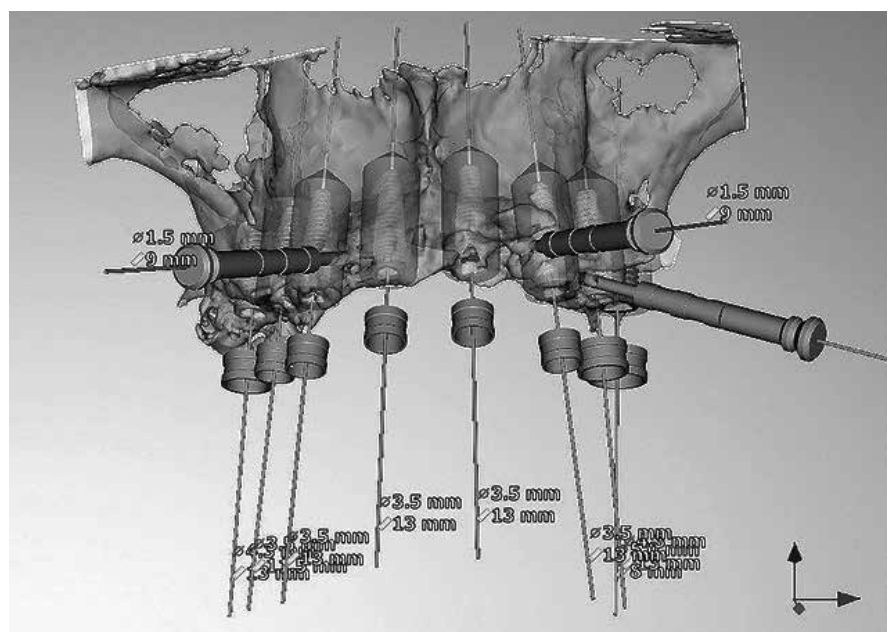

Figura 5. Planificación quirúrgica con software Nobelguide ${ }^{\circledR}$ de imagen de CBCT.

mente y con el uso de un software de planificación, se puede optimizar su ubicación antes de la cirugía 17. Así, los implantes pueden ser planificados para soportar una prótesis que proporcione los requisitos 
biológicos, funcionales y estéticos ideales y al mismo tiempo respetar la anatomía maxilar o mandíbular obtenida por las imágenes de la CBCT. Esta técnica puede, por supuesto, contribuir para un éxito a largo plazo de la rehabilitación con estos implantes. En este sentido, una revisión sistemática indicó una tasa media de éxito del 97,3\%, utilizando la técnica de cirugía guiada y después de un período de seguimiento de al menos 12 meses ${ }^{18}$.

Además, se puede prever la necesidad de regeneración ósea, así como de modificaciones protésicas (p. ej., la utilización de pilares angulados). De este modo es más fácil conseguir un resultado estético predecible, una vez que las posiciones planificadas de los implantes se transfieren de una forma más precisa al ambiente quirúrgico mediante la férula guiada $^{16}$.

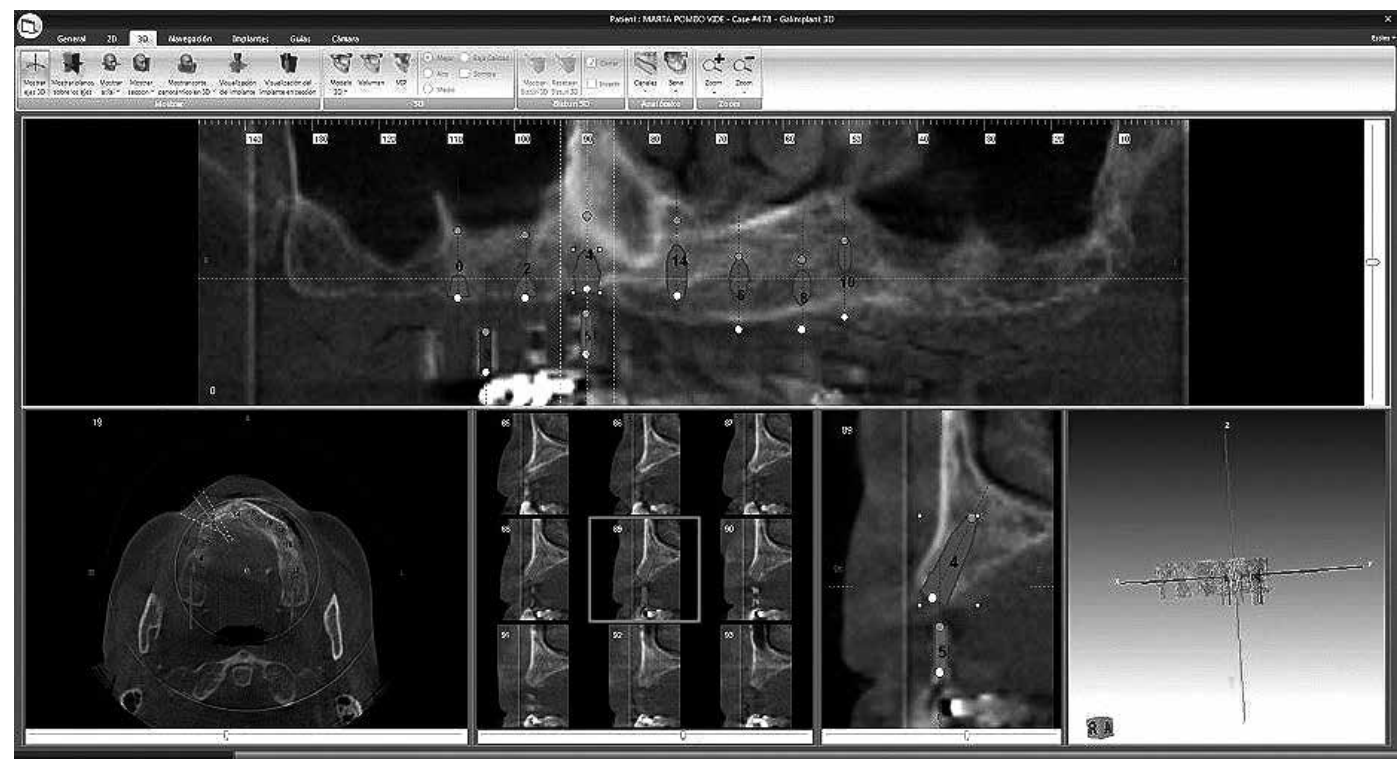

Figura 6. Planificación quirúrgica con software Galimplant $3 \mathrm{D}{ }^{\circledR}$ de imagen de CBCT.

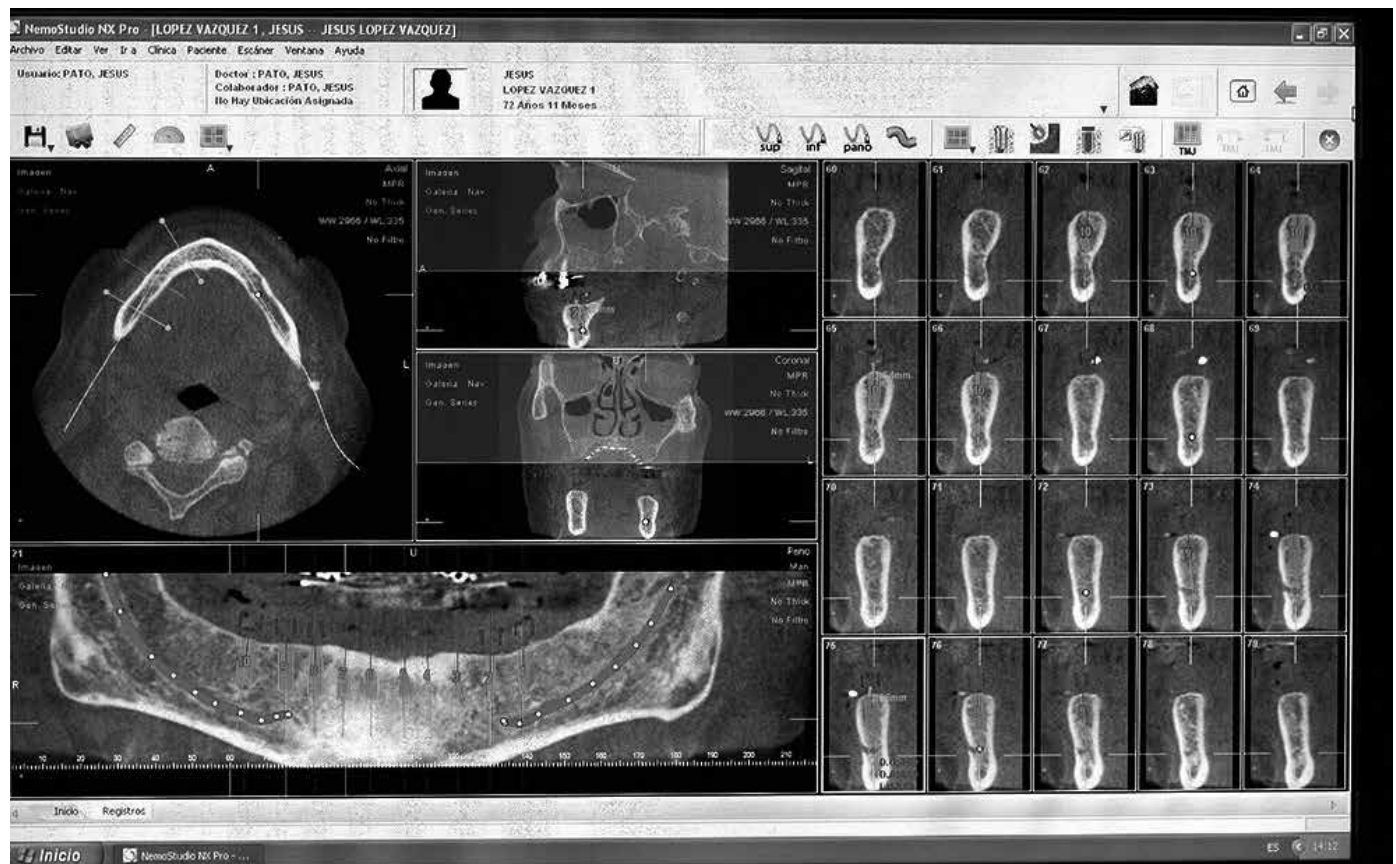

Figura 7. Planificación quirúrgica virtual con software ${ }^{\circledR}$ de imagen de CBCT.

La duración de una intervención quirúrgica puede influir negativamente en la morbilidad del paciente. En un estudio compararon la duración de la colocación de los implantes en pacientes edéntulos totales (una cirugía guiada sin colgajo versus una cirugía guiada con colgajo versus una cirugía convencional) y reportaron que la cirugía sin colgajo tomó la mitad del tiempo de las otras dos cirugías ( $24 \mathrm{~min}$. en la segunda vs. más de 60 min en la tercera) ${ }^{19}$. En otro estudio similar, los mismos investigadores compararan la cirugía convencional $(n=29)$ con la cirugía guiada por ordenador $(n=34)$ en pacientes totalmente edéntulos, y reportaron de nuevo una reducción significativa en el tiempo quirúrgico para la opción guiada (63 vs 33 min, respectivamente) 20 .

La colocación guiada de los implantes también facilita los tratamientos que implican la confección de una restauración inmediata ${ }^{21}$. Incluso permite el uso de una restauración que se puede colocar 


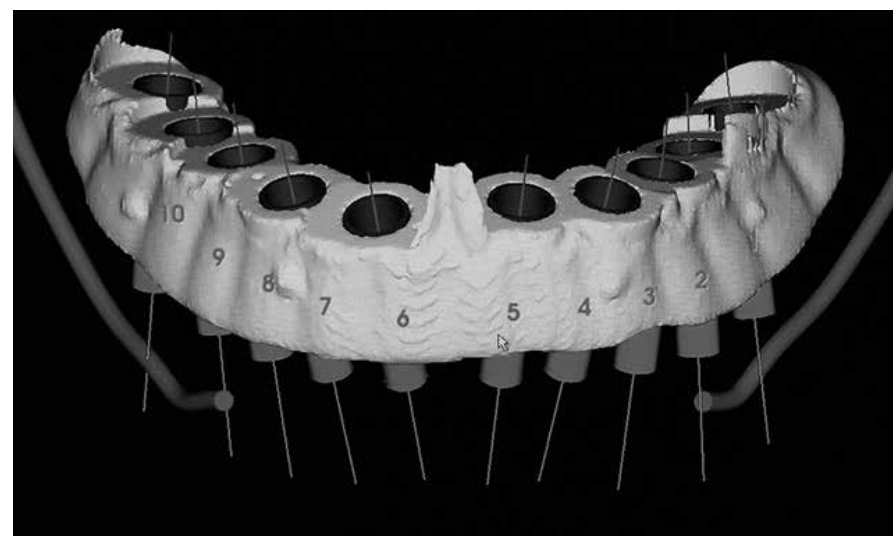

Figura 8. Elaboración virtual de férula quirúrgica guiada.

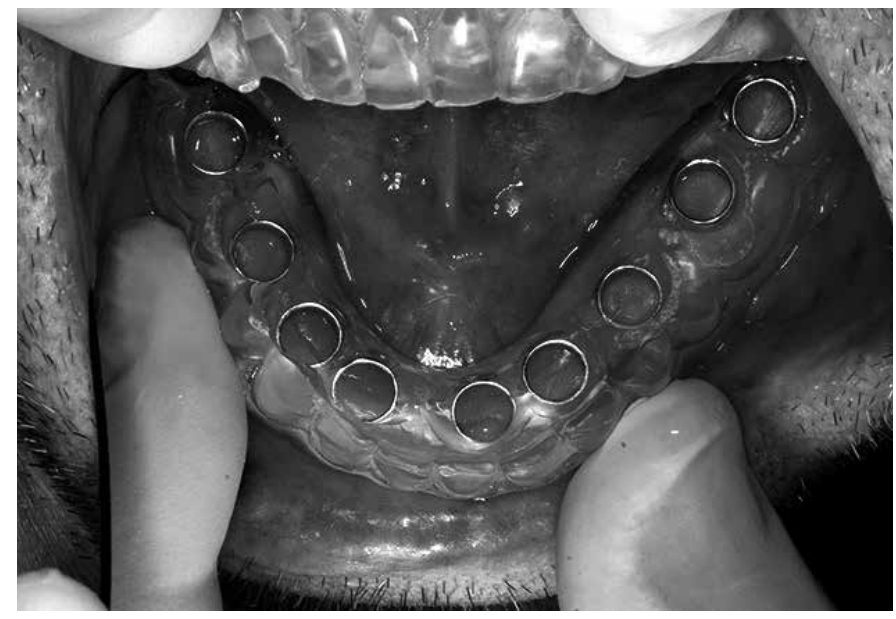

Figura 9. Prueba de posicionamiento de la férula quirúrgica guiada.

inmediatamente después de la cirugía, mejorando la satisfacción del paciente, así como su comodidad, función, y estética ${ }^{21-22}$. La aplicación de restauraciones inmediatas está asociada a tasas de supervivencia aceptables de los implantes, que oscilan entre el $91 \%$ y el $100 \%{ }^{22}$.

Es interesante saber si la cirugía guiada se puede emplear como alternativa a las técnicas de aumento óseo. En este sentido, se ha utilizado la técnica de cirugía guiada en casos de pacientes parcialmente edéntulos con el maxilar seriamente reabsorbido y han obtenido una tasa de supervivencia de los implantes de $98 \%$ a los 4 años, sin cualquier procedimiento del aumento, lo que sugiere que en determinados casos, la cirugía guiada se puede utilizar como una alternativa para las técnicas de aumento óseo ${ }^{23}$.

La cirugía guiada representa una alternativa de tratamiento excelente para pacientes con cantidad ósea satisfactoria para la inserción de implan- tes, en pacientes completamente edéntulos en el maxilar o en la mandíbula ${ }^{24}$. Es la mejor opción de tratamiento para el paciente con condiciones médicas comprometidas, como la reconstrucción mandibular después de una resección oncológica, o pacientes geriátricos. Aunque la cirugía sin colgajo es ventajosa para la colocación del implante, requiere tejido blando suficiente y una cantidad ósea adecuada. Para los casos con una encía muy delgada, la cirugía guiada es útil, pero cuando es asociada a una técnica con colgajo ${ }^{24}$.

El éxito de una prótesis de carga inmediata depende de la exactitud de todos los pasos clínicos y de laboratorio implicados en la planificación virtual ${ }^{16-17}$. El éxito final es influenciado por el mantenimiento de la prótesis inmediata durante el período de cicatrización hasta la colocación de la prótesis definitiva ${ }^{25}$. La utilización solamente de la planificación asistida por ordenador sin hacer cirugía guiada podrá también ser beneficioso para el paciente y el clínico ${ }^{16}$.

Aunque el beneficio clínico de la cirugía guiada es cuestionable para algunos autores, existe un consenso general sobre sus ventajas, como la posibilidad de hacer una planificación quirúrgica virtual, el procedimiento quirúrgico facilitado, el menor tiempo de intervención y la menor morbilidad ${ }^{26}$. Los distintos sistemas de cirugía guiada incorporan la planificación de la colocación del implante, utilizando una variedad de herramientas de software $^{26}$.

Se han categorizado estos sistemas informáticos para cirugía guiada en estáticos y dinámicos. Los sistemas estáticos son aquellos que comunican las posiciones predeterminadas de los implantes al campo operatorio usando férulas quirúrgicas, mientras los sistemas dinámicos comunican las posiciones de los implantes seleccionados al campo operatorio con herramientas de imagen visuales en un monitor de un ordenador, en lugar de férulas intraorales rígidas ${ }^{6,27}$. Los sistemas dinámicos incluyen la navegación quirúrgica y las tecnologías de navegación asistida por ordenador, permitiendo al cirujano alterar el procedimiento quirúrgico y la posición del implante en tiempo real, utilizando la información anatómica disponible del plan preoperatorio y una CBCT ${ }^{16}$. Una vez que el cirujano puede ver una representación 3D del fresado en relación con la anatomía del paciente durante la cirugía, las modificaciones se 


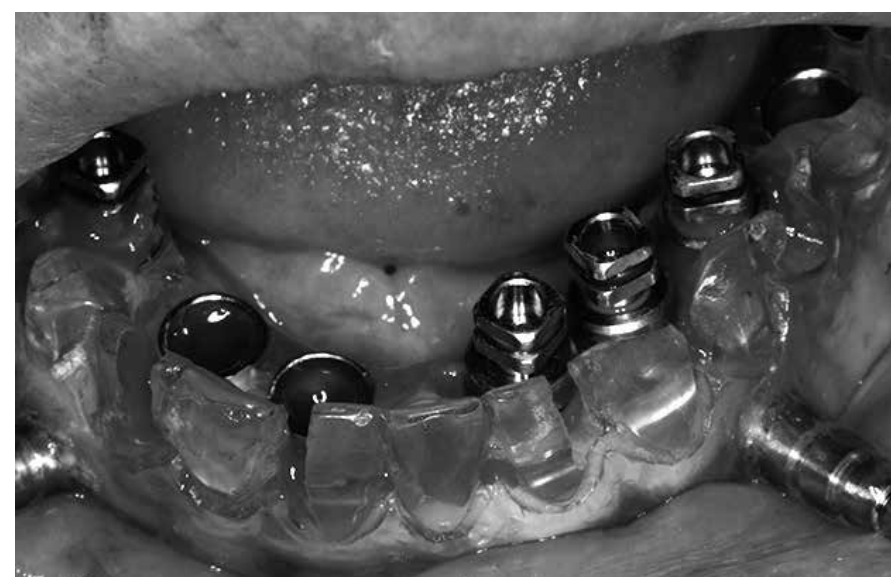

Figura 10. Inserción de los implantes con la férula quirúrgica guiada.

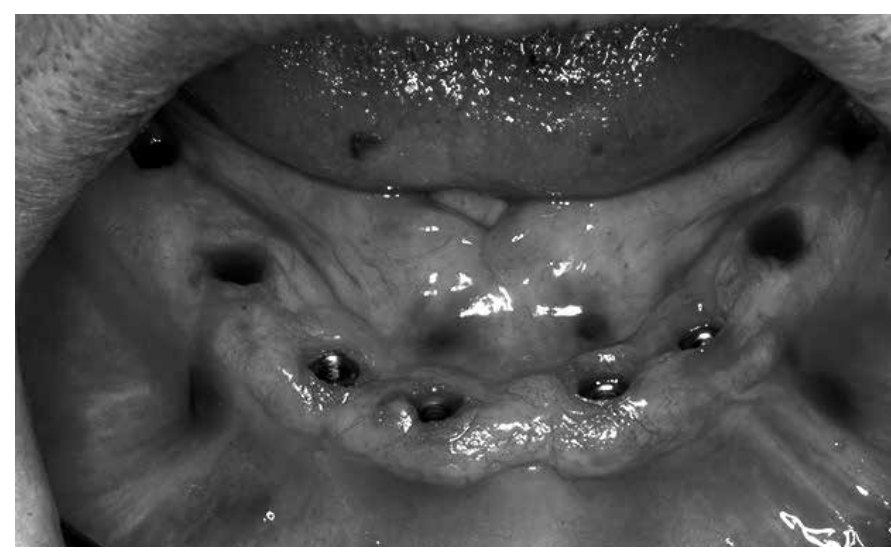

Figura 11. Cirugía finalizada de la inserción de los implantes con la férula quirúrgica guiada.

pueden lograr con más facilidad. En esencia, el enfoque dinámico proporciona una guía quirúrgica virtual que puede ser alterada según las condiciones encontradas durante la cirugía.

Se ha afirmado los sistemas estáticos tienen la tendencia a ser más precisos que los sistemas dinámicos. Sin embargo, la mayoría de los artículos publicados sobre cirugía dinámica han sido sobretodo estudios clínicos, mientras que la mayoría de los protocolos estáticos estudiados han sido preclínicos (en modelos o en cadáver), donde se pueden realizar mediciones más precisas ${ }^{18}$. La mayor precisión de estos últimos estudios se puede explicar por el mejor acceso, el mejor control visual del eje de la osteotomía, la falta de movimiento en el cadáver, y la ausencia de saliva o sangre en los modelos preclínicos. En los sistemas dinámicos, la osteotomía y la inserción del implante se pueden alterar durante la cirugía. Así la osteotomía no tiene ninguna otra férula quirúrgica que la visión del cirujano en el modelo virtual, dándose la capa- cidad de elegir la posición del implante basada en la evaluación de la anatomía del lecho implantar, en tiempo real ${ }^{16}$.

Los diversos sistemas de cirugía guiada por ordenador presentan distintas características, como por ejemplo el diseño de las fresas utilizadas en la osteotomía. Algunos sistemas tienen fresas con topes y otros sistemas tienen fresas con líneas indicadoras, para controlar la profundidad del fresado. Algunos sistemas utilizan, para el mismo paciente, más de una férula quirúrgica, con conductos metálicos de un diámetro creciente, mientras que otros sistemas usan una sola férula quirúrgica, y utilizan en los conductos metálicos las guías de fresa de diámetros diferentes. Algunos sistemas permiten una inserción totalmente guiada del implante, mientras que otros sistemas, su férula quirúrgica sólo sirve para que la fresa piloto guiada oriente la dirección del fresado. A continuación la férula es removida y la inserción de los implantes se procede de una manera convencional ${ }^{16,28}$.

No suele haber diferencias significativas en la precisión de la técnica de cirugía guiada en la mandíbula en comparación con el maxilar ${ }^{16,18}$. La importancia de una desviación de la precisión de esta técnica depende de las condiciones anatómicas de la zona elegida para operar (el volumen óseo disponible, la presencia de estructuras neurológicas que tienen que ser preservadas), de la distancia entre los implantes y los dientes, y de la configuración de la futura rehabilitación protésica. La experiencia clínica existente sobre este tema reporta que, genéricamente, es aceptable una desviación en el error de precisión de $1,5 \mathrm{~mm}$, un valor menor que lo de la cirugía no guiada ${ }^{16}$.

\section{SOFTWARES DE PLANIFICACION DE CIRUGÍA GUIADA}

En esta técnica es importante seguir una serie de pasos clínicos en el protocolo, tener en cuenta algunas consideraciones radiológicas y realizar una correcta planificación, todo ello va a influir directamente en el acto quirúrgico. En la cirugía guiada asistida por ordenador, la posición y la inclinación de los implantes son una suma de información de los datos obtenidos de la tomografía computarizada y de la planificación de los mismos en el sof- 
tware informático (Figuras 3-6). La transferencia de la planificación de la rehabilitación al campo operatorio, se realiza mediante una guía quirúrgica elaborada con un proceso de protocolo rápido y además en muchas ocasiones permitiendo realizar carga inmediata con la colocación de una rehabilitación fija provisional (Figuras 7-11) ${ }^{29}$.

Los componentes necesarios para preparar una cirugía guiada de implantes dentales son el conjunto de datos analizados de las imágenes de la CBCT, el software de planificación quirúrgica, la férula radiológica y la férula quirúrgica. Las características de estos dos últimos componentes dependen en gran medida del software elegido. El software de planificación de implantes permite planificar virtualmente la cirugía de implantes y diseñar férulas quirúrgicas a través de la información adquirida (Figuras 7-11). Una correcta férula quirúrgica es la que permite al clínico colocar con precisión los implantes en la posición deseada, con una trayectoria de inserción predefinida, con una tolerancia mínima, no flexible, y permanecer estable durante el procedimiento quirúrgico (Figuras $9-11)^{29,30}$.

Actualmente, existen diversos programas de software de planificación de implantes, y algunos sistemas que proporcionan una planificación usando el software nativo de las unidades de adquisición de la CBCT (Figuras 3-6). Después de esta adquisición, las imágenes se exportan a los archivos DICOM, un estándar para la distribución y visualización de imágenes médicas, independientemente de su origen. Cuando el conjunto de datos se carga en el software los datos deben ser interpretados de una forma completa. Una reconstrucción de la CBCT se obtiene de todas las imágenes creadas y permite una visualización con diferentes perspectivas. Por lo tanto, una sección transversal, una vista panorámica, o una vista multiplanar, entre otros, son considerados reconstrucciones de la CBCT. Para propósitos de planificación de implantes, la sección transversal es la imagen de diagnóstico primaria utilizada para la evaluación del volumen y la calidad ósea. Sin embargo, usando el principio de la correlación, muchas otras reconstrucciones pueden y deben ser utilizadas para mejorar el proceso diagnóstico ${ }^{9-12}$.

Todos los programas de software permiten la visualización del conjunto de datos en la vista de reconstrucción multiplanar, que es el modo de visualización predeterminado en CBCT (Figuras 3-6). Las imágenes 2D se pueden visualizar en 3 planos diferentes (axial, coronal y sagital). Algunos programas de software incluyen una imagen $3 \mathrm{D}$ que es un producto final del procesamiento digital. La información sobre los 3 planos diferentes debe ser completamente interpretada, porque cada plano proporciona información diferente de las diferentes estructuras incluidas en el campo de visión (FOV) ${ }^{9-12}$.

La mayoría de los softwares de imágenes de la CBCT permiten al implantólogo manipular las imágenes con controles flexibles y variados. Esta flexibilidad no sólo proporciona una gran cantidad de creatividad y potencial de generación de imágenes, sino que también puede llevar a errores de reconstrucción de imágenes múltiples, si los principios específicos no se establecen como un estándar. La mayoría de estos principios se refieren a cómo se orientan los datos de volumen y cómo se crean imágenes de corte transversal. Hacer esto incorrectamente resulta en imágenes que pueden conducir a errores visuales y de medida ${ }^{30}$.

Una vez efectuadas las mediciones en las imágenes transversales, el clínico puede colocar los implantes en las regiones de interés con las características obtenidas a partir de las mediciones preliminares $^{1,3-4}$. Los softwares de planificación de implantes incluyen bibliotecas con la mayoría de los implantes disponibles en el mercado y sus pilares compatibles (tanto rectos como angulados). Estas bibliotecas se actualizan constantemente. La mayoría de los softwares permiten la creación de una zona de seguridad alrededor del cuerpo del implante virtual (que puede ajustarse a 1-2 mm) ayudando a minimizar la invasión del espacio biológico por errores de manipulación de datos. Los perfiles de emergencia también pueden ser identificados usando las proyecciones de los pilares. Se pueden colocar formas de dientes virtuales para la simulación de la corona final (cementada o atornillada). Estos dientes virtuales se pueden individualizar para cada paciente, permitiendo la simulación de las necesidades protésicas de la zona a rehabilitar ${ }^{30}$.

\section{CONCLUSIONES}

En la cirugía guiada asistida por ordenador, las imágenes radiológicas obtenidas por la CBCT, son 
procesadas por un software que permite realizar una planificación con la inserción de los implantes de forma virtual. Estas nuevas técnicas digitales de imagen 3D, permiten evaluar la selección de las localizaciones quirúrgicas de los implantes en un modelo virtual, según las características anatómicas del maxilar y/o mandíbula edéntulos y establecer un protocolo clínico, quirúrgico y prostodóncico, más favorable para la rehabilitación funcional y estética del paciente.

\section{BIBLIOGRAFIA}

1. Lorrio JM, Sierra L, García I, Lorrio C, Gómez R. La rehabilitación con implantes en el paciente edéntulo maxilar mediante cirugía guiada y carga inmediata. Av Periodon Implantol 2015; 27: 117-124.

2. Joda T, Ferrari M, Gallucci GO, Wittneben JG, Brägger U. Digital technology in fixed implant prosthodontics. Periodontol 2000 2017;73:178-92.

3. Lanis A, Del Canto O. The combination of digital surface scanners and cone beam computed tomography technology for guided implant surgery using 3shape implant studio software: a case history report. Int J Prosthodont 2015;28:169-78.

4. Marchack CB, Chew LK. The 10-year evolution of guided surgery. J Calif Dent Assoc 2015;43:131-4.

5. Colombo M, Mangano C, Mijiritsky E, Krebs $M$, Hauschild U, Fortin T. Clinical applications and effectiveness of guided implant surgery: a critical review based on randomized controlled trials. BMC Oral Health 2017; 17:150.

6. d'Haese J, Ackhurst J, Wismeijer D, de Bruyn $\mathrm{H}$, Tahmaseb A. Current state of the art of computer-guided implant surgery. Periodontology 2000 2017; 73:121-133.

7. Pyo SW, Lim YJ, Koo KT, Lee J. Methods used to assess the 3D accuracy of dental implant positions in computer-guided implant placement: a review. J. Clin Med 2019; 8: 54.

8. Bernard L, Vercruyssen M, Duyck J, Jacobs R,
Teughels W, Quirynen M. A randomized controlled clinical trial comparing guided with nonguided implant placement: A 3-year follow-up of implant-centered outcomes. J Prosthet Dent 2019: S0022-3913(18)30766-2.

9. Chan HL, Misch K, Wang HL. Dental imaging in implant treatment planning. Implant Dent 2010;19:288-98.

10. Harcher DC. Operational principles for cone-beam computed tomography. JADA 2010; 141(suppl): 3S-6S.

11. Pedroso L, Garcia R, Leles J, Leles C, Silva M. Impact of cone-beam computed tomography on implant planning and on prediction of implant size. Brazilian Oral Res 2014;28:46-53.

12. Zhang W, Skrypczak A, Weltman R. Anterior maxilla alveolar ridge dimension and morphology measurement by cone beam computerized tomography (CBCT) for immediate implant treatment planning. BMC Oral Health 2015; 15:65.

13. Shelley AM, Ferrero A, Brunton P, Goodwin M, Horner K. The impact of CBCT imaging when placing dental implants in the anterior edentulous mandible: a before-after study. Br Dent $\mathrm{J}$ 2018; 224:601-11.

14. Liu J, Chen HY, Dodo H, Yousef H, Firestone A, Chaudry J, et al. Efficacy of cone-beam computed tomography in evaluating bone quality for optimum implant treatment planning. Impl Dent 2017; 26:405-11.

15. Pommer B, Busenlechner $D$, Fürhauser $R$, Watzek G, Mailath-Pokorny G, Haas R. Trends in techniques to avoid bone augmentation surgery: Application of short implants, narrow-diameter implants and guided surgery. J CranioMaxillofacial Surg 2016;44:1630-4.

16. Vercruyssen M, Laleman I, Jacobs R, Quirynen $M$. Computer-supported implant planning and guided surgery: a narrative review. Clin Oral Implants Res 2015;26(suppl.1):69-76.

17. Scherer MD. Presurgical implant-site assessment and restoratively driven digital planning. Dent Clin North Am. 2014;58:561-95. 
18. Tahmaseb A, Wismeijer D, Coucke W, Derksen W. Computer technology applications in surgical implant dentistry: a systematic review. Int J Oral Maxillofac Implant. 2014;29(suppl.):25-42

19. Arisan V, Karabuda ZC, Ozdemir T. Accuracy of two stereolithographic guide systems for computer-aided implant placement: a computed tomography-based clinical comparative study. J Periodontol 2010;81:43-51.

20. Arısan V, Karabuda CZ, Mumcu E, Özdemir $\mathrm{T}$. Implant positioning errors in freehand and computer-aided placement methods: a single-blind clinical comparative study. Int J Oral Maxillofac Implants 2013;28:190-204.

21. Vandenberghe B. The digital patient - Imaging science in dentistry. J Dent 2018;74:21-6.

22. Pozzi A, Tallarico M, Marchetti M, Scarfò B, Esposito M. Computer-guided versus freehand placement of immediately loaded dental implants: 1-year post-loading results of a multicentre randomised controlled trial. Eur $\mathrm{J}$ Oral Implant 2014;7:229-42.

23. Fortin T, Isidori $M$, Bouchet $H$. Placement of posterior maxillary implants in partially edentulous patients with severe bone deficiency using CAD/CAM guidance to avoid sinus grafting: a clinical report of procedure. Int $\mathrm{J}$ Oral Maxillofac Implants 2009;24:96-102.

24. Velasco-Ortega E, Matos-Garrido N, Ortiz-García I, España-López A, Jiménez-Guerra A, Moreno-Muñoz J, Núñez-Márquez E. Carga precoz con sobredentadura mandibular sobre dos implantes insertados mediante cirugía guiada. Av Odontoestomatol 2017; 33:231-8.
25. Drago C, del Castillo R, Peterson T. Immediate occlusal loading in edentulous jaws, CT-guided surgery and fixed provisional prosthesis: a maxillary arch clinical report. J Prosthodont $2011 ; 20: 209-17$.

26. Kühl S, Payer M, Zitzmann NU, Lambrecht JT, Filippi A. Technical accuracy of printed surgical templates for guided implant surgery with the coDiagnostiXTM software. Clin Implant Dent Relat Res 2015;17(suppl.1):177-82.

27. Jung RE, Schneider D, Ganeles J, Wismeijer D, Zwahlen M, Hämmerle C, et al. Computer technology applications in surgical implant dentistry: a systematic review. Int J Oral Maxillofac Implants. 2009;24(suppl.): 92-109.

28. Younes F, Cosyn J, De Bruyckere T, Cleymaet R, Bouckaert E, Eghbali A. A randomized controlled study on the accuracy of free-handed, pilot-drill guided and fully guided implant surgery in partially edentulous patients. J Clin Periodontol 2018;45:721-32.

29. Katleen Bragança L, Pato Mourelo J, Díaz Prada FJ, López Castro JJ, Anache A. Rehabilitación fija maxilar con implantes mediante cirugía guiada asistida por ordenador. Av Odontoestomatol 2017; 33: 197-203.

30. Mora MA, Chenin D, Arce R. Software tools and surgical guides in dental-implant-guided surgery. Dent Clin North Am. 2014;58(3):597-626.

\section{AUTOR DE CORRESPONDENCIA:}

Prof. Eugenio Velasco Ortega

Facultad de Odontología

C/ Avicena s/n Tfno: 954481132

email: evelasco@us.es

41009 Sevilla 